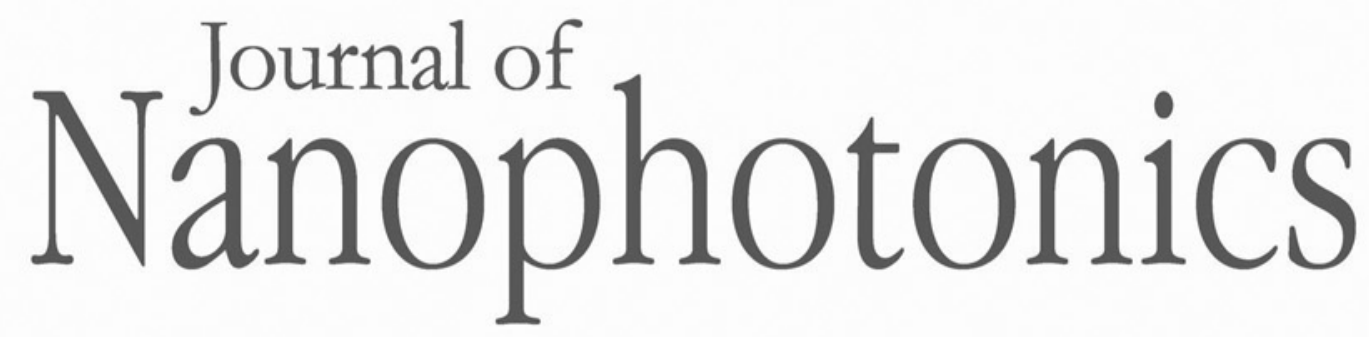

Nanophotonics.SPIEDigitalLibrary.org

\title{
Spatially relocatable and spectrally tunable photonic crystal cavity by using a microsphere
}

\author{
Young Jin Lee \\ Tae-Woo Lee \\ Da Eun Lee \\ Seokhyeon Hong \\ Soon-Hong Kwon
}




\title{
Spatially relocatable and spectrally tunable photonic crystal cavity by using a microsphere
}

\author{
Young Jin Lee, Tae-Woo Lee, Da Eun Lee, \\ Seokhyeon Hong, and Soon-Hong Kwon* \\ Chung-Ang University, Department of Physics, 84, Heukseok-ro, Dongjak-gu, \\ Seoul 06974, Republic of Korea
}

\begin{abstract}
We proposed a spectrally tunable photonic crystal (PC) cavity that is also relocatable spatially. The PC cavity is formed by setting a microsized dielectric sphere on a defect-free PC slab at a desired point. The resonant wavelength of the PC cavity can be tuned from 1343 to $1725 \mathrm{~nm}$ by changing the parameters of the sphere, e.g., the radius or the refractive index. In addition, the wavelength is also adjusted finely $(\sim 5 \mathrm{~nm})$ by moving the sphere upward. $\odot$ The Authors. Published by SPIE under a Creative Commons Attribution 3.0 Unported License. Distribution or reproduction of this work in whole or in part requires full attribution of the original publication, including its DOI. [DOI: 10.1117/1.JNP.10.030501]
\end{abstract}

Keywords: photonic crystal cavity; spatially relocatable cavity; spectrally tunable cavity.

Paper 16090L received Jun. 7, 2016; accepted for publication Jul. 22, 2016; published online Aug. 9, 2016.

\section{Introduction}

Photonic crystals (PCs) are receiving attention because of their attractive optical properties. PCs are composed of a periodic array of dielectric mediums with different refractive indices; they can manipulate the propagation of lights with a wavelength-scale structure. PC cavities have been applied to nanolasers, ${ }^{1-6}$ optical switches, ${ }^{7,8}$ and single-photon sources ${ }^{9-14}$ because of their low optical loss and wavelength-sized mode volume, which enables strong light-matter interactions. In particular, the single-photon sources employed for quantum cryptology consist of a high quality factor $(Q)$ PC cavity and a quantum dot (QD) as an atom-like emitter. When the QD is placed at the antinode of the cavity-mode profile and the emission wavelength of the QD is matched with the cavity resonance, the light-matter interactions are maximized and the system of a QDPC cavity can play the role of an efficient single-photon source. However, since a PC cavity is fabricated by a semiconductor process such as e-beam lithography and etching, ${ }^{2,15}$ it is not guaranteed that the determined characteristics of the PC cavity, resonant wavelength, and mode profile match the emission wavelength and the position of a QD grown randomly in the wafer. In fact, the operation of a QD-based PC cavity as the single-photon source usually depends on accidentally matching the spectral/spatial position of the QD and the cavity, which makes an applicable single-photon source a very challenging and impractical device. Therefore, a PC cavity that has spatial position flexibility and spectral tenability would be an important milestone for the development of a useful single-photon source. On the other hand, a spatially tunable PC cavity has been reported in which a bent tapered optical fiber is brought close to a PC to form a cavity, while having a limit of mechanically weakness of tapered fiber. ${ }^{16}$

In our research, we propose a PC cavity that is spatially relocatable and spectrally tunable and analyze properties of the structure by calculating three-dimensional model with finite-differential time-domain method. The proposed PC cavity is composed of a microscale dielectric sphere on a defect-free PC slab. The position of the PC cavity is relocated to a desired point simply by moving the sphere to that point. A resonant wavelength of the PC cavity is also tuned, not only coarsely by modifying parameters of the sphere (e.g., the radius and the refractive

*Address all correspondence to: Soon-Hong Kwon, E-mail: soonhong.kwon@gmail.com 
index) but also finely by changing the height of the sphere from the PC slab. The single-photon source is simply formed by using the attributes of the proposed PC cavity.

\section{Spatial Relocatability}

When a microsphere is placed on the PC slab, the effective refractive index near the sphere increases and a localized defect is formed in the PC slab, which works as a PC cavity. The PC composed of a triangular lattice has a periodicity $(a)$ of $550 \mathrm{~nm}$ and an air-hole radius of $192.5 \mathrm{~nm}(0.35 a)$. The slab thickness is $200 \mathrm{~nm}$. The PC slab has a photonic band structure as shown in Fig. 1(b). In the PC structure, the photonic bandgap is from 1309 to $1744 \mathrm{~nm}$. In this study, the investigated radius of the sphere ranges from 1000 to $1600 \mathrm{~nm}$ and the refractive index of the sphere is assumed to range from 1.70 to 3.40 .

The fabrication of the usual PC cavities needs to be state-of-art e-beam lithography or semiconductor etching for patterning a defect in the PC. For example, in an air-hole PC slab, a defect is formed by reducing or removing an air hole because the effective refractive index of the modified hole increases. However, the proposed cavity is formed when a dielectric sphere contacts a defect-free PC slab. In the cavity, no physical defect exists in the PC slab; however, the local index increases due to the existence of a microsphere working like a defect. Therefore, the proposed cavity only needs a noncomplex method such as laser holography for patterning a defectfree PC slab. ${ }^{17,18}$ In addition, the position and resonant wavelength of the cavity are variable because of their dependency on the parameters of the sphere, such as the position, radius, and refractive index.

In order to figure out the cavity modes that are formed by the position of the dielectric microsphere, we investigate the typical positions of the triangular lattice, as shown in Figs. 2(a) and 2(c). First, a microsphere with a radius of $1500 \mathrm{~nm}$ and a refractive index of 1.95 is placed on a black spot in Fig. 2(a), which is at the center of the three air holes creating the triangular lattice. This cavity, called a zero-cell cavity, ${ }^{5}$ has three localized cavity modes, such as a single monopole mode and doubly degenerated dipole modes, as shown in Fig. 2(b). One dipole mode has symmetry along the $x$ axis, another has symmetry along the $y$ axis. The resonant wavelengths are $1477 \mathrm{~nm}$ for the monopole mode and $1476 \mathrm{~nm}$ for the degenerated dipole modes. All three modes are strongly localized near the contact position of the microsphere. The $Q$ factors are 480 and 380 for the monopole and dipole modes, respectively.

On the other hand, the PC cavity mode can also be excited when a dielectric microsphere with a radius of $1500 \mathrm{~nm}$ and an index of 2.35 is placed at the center of an air hole, as indicated by the black dot in Fig. 2(c). In contrast to this, the sphere makes contact with the surface of the slab in Figs. 2(a) and 2(b), and the sphere is partially inserted inside an air hole (inset). The cavity supports the same PC cavity modes with a single-cell cavity ${ }^{1,3,19}$ and has doubly degenerated

(a)

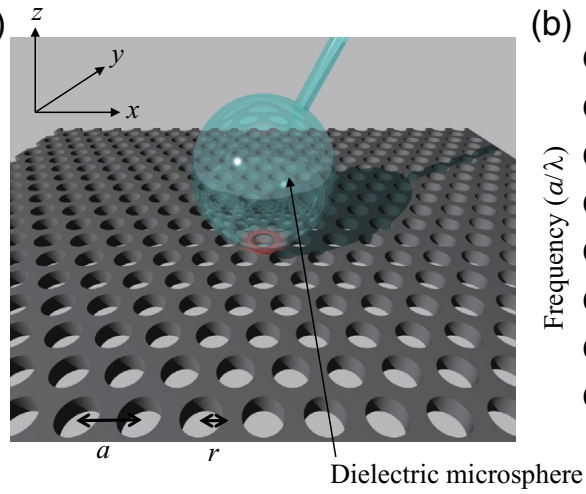

(b)

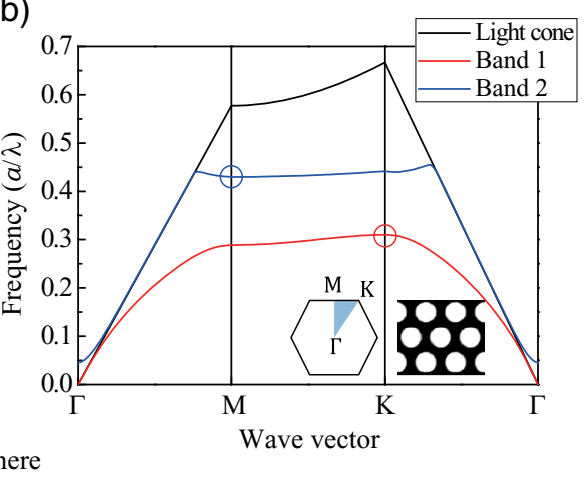

Fig. 1 (a) Scheme of a tunable PC cavity with a dielectric microsphere and a two-dimensional (2-D) triangular lattice PC slab. The slab index is assumed to be 3.4. Thickness $(t)$, lattice constant (a), and air-hole radius $(r)$ are 200,550 , and $192.5 \mathrm{~nm}$, respectively. (b) Photonic band structure of the PC slab. The bandgap is formed from 1309 (blue circle) to $1774 \mathrm{~nm}$ (red circle). 


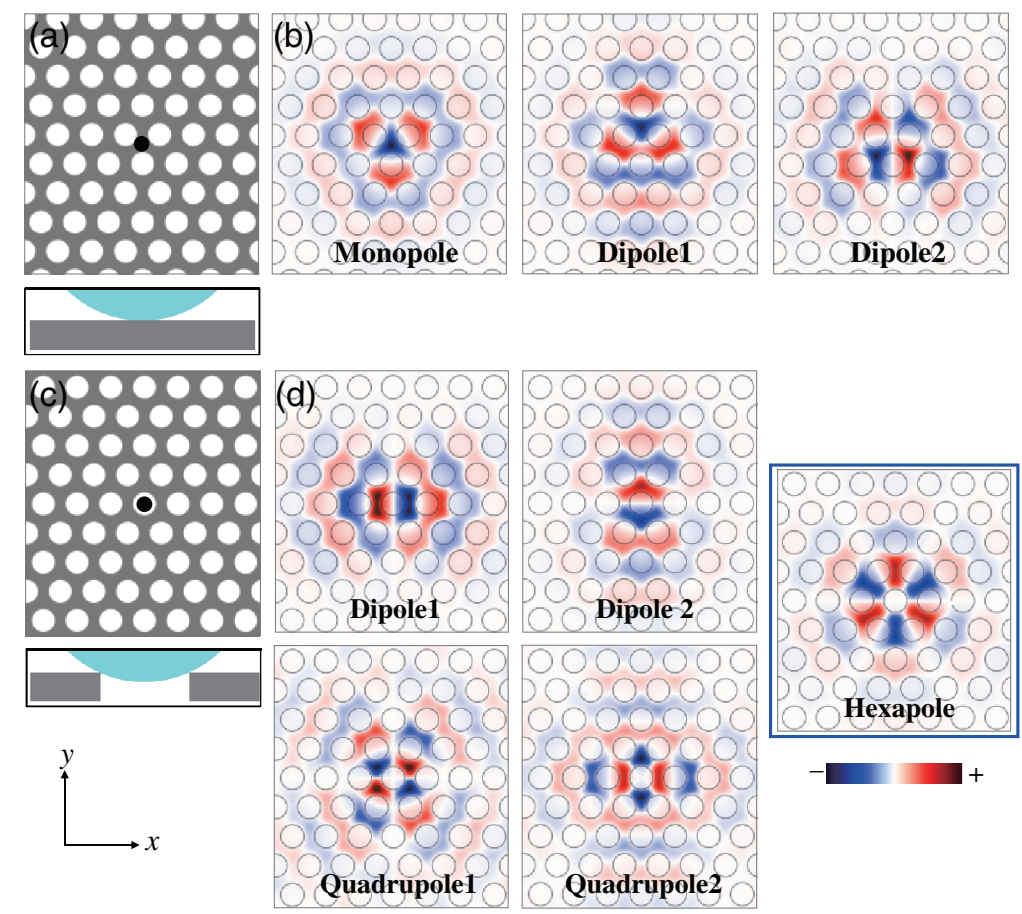

Fig. 2 (a) Microsphere position between the air holes. (b) Mode profiles of tunable PC cavity modes, monopole, dipole 1 , and dipole 2 when a microsphere is placed on the black dot in (a). The microsphere has a refractive index, $n_{\text {sphere }}=1.95$ and a radius $R=1500 \mathrm{~nm}$. (c) Microsphere position at the center of the air hole. (d) Mode profiles of the PC cavity modes, double-degenerated dipoles and quadrupoles, and hexapole when a microsphere is placed on the black dot in (c). The microsphere has a refractive index $n_{\text {sphere }}=2.35$ and a radius $R=1500 \mathrm{~nm}$.

dipole modes, doubly degenerated quadrupole modes, and a single hexapole mode as shown in the mode profiles (Hz field) of Fig. 2(d). The resonant wavelengths are $1616 \mathrm{~nm}$ for the dipole modes, $1310 \mathrm{~nm}$ for the quadrupole modes, and $1496 \mathrm{~nm}$ for the hexapole mode. Since the frequency is placed outside the bandgap, the monopole mode is not observed. The $Q$ factors are 10,000 for the dipole modes, 1,000 for the quadrupole modes, and 6,800 for the hexapole mode. The hexapole mode has a high $Q$ factor due to mode cancellation. ${ }^{20,21}$ Here, the refractive indices, 1.95 (e.g., index of $\mathrm{SiN}$ ) and 2.35 (e.g., index of $\mathrm{TiO}_{2}$ ), of the spheres in Figs. 2(a) and 2(c) are chosen for the all presented cavity modes to be well-localized horizontally.

Therefore, the cavity mode of the proposed cavity at an arbitrary point is created by setting the sphere on the point. Consequently, the cavity mode is well formed wherever the sphere comes between the air holes or when it is at the center of an air hole with a high $Q$ factor and wavelength-sized mode volume. In other words, the position of the cavity is relocatable spatially by adjusting one of the spheres.

The hexapole mode still has characteristics of the general single-cell mode, e.g., a large $Q$ value and small mode volume. However, unlike the general single-cell hexapole mode, the hexapole mode created by a sphere can have several resonant wavelengths with an individual azimuthal mode number in the sphere at the $Y Z$-plane, as shown in Fig. 3. For example, the resonance of a hexapole mode is observed at 1357, 1463, 1591, and $1748 \mathrm{~nm}$ in the bandgap when the sphere with a 1000-nm radius and 2.91-refractive index makes contact with the slab. Each mode shows the field at the edge of the sphere like the whispering guided mode, ${ }^{22-24}$ as shown in the mode profile of the cross-section of the $Y Z$-plane [Fig. 3(b)]. The modes can be classified by the number of oscillations in the circumference of the sphere, called the azimuthal mode number. In this case, the azimuthal mode numbers of each mode are 9, 8, 7, and 6 . Therefore, the proposed cavity is free to choose the resonant wavelength when the cavity is coupled with an emitter because the resonant wavelength is selected in a large region from 


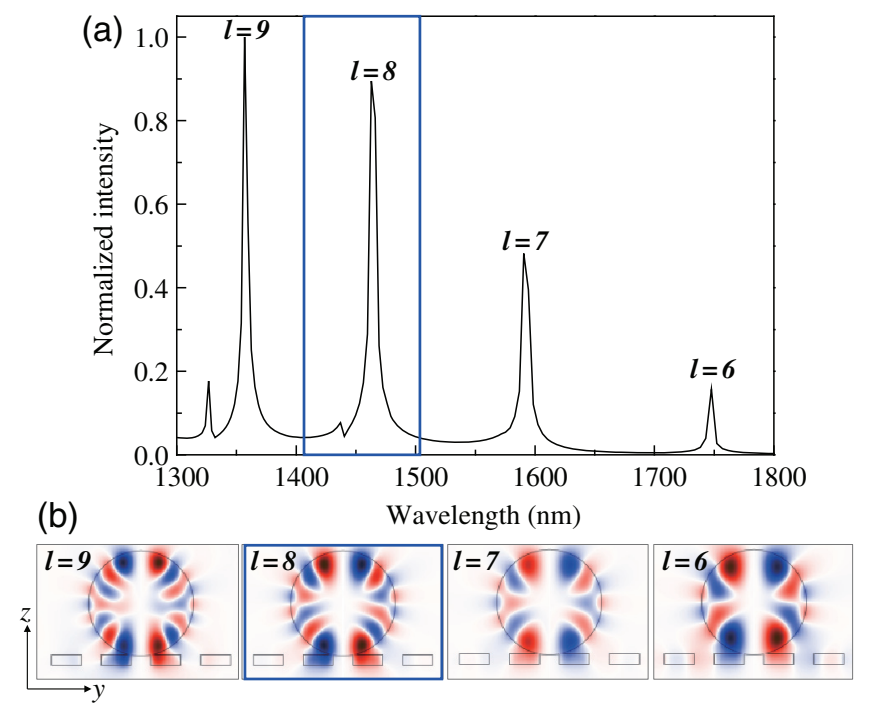

Fig. 3 (a) Spectrum of hexapole modes when the radius and the refractive index of the microsphere are $1000 \mathrm{~nm}$ and 2.91, respectively. The resonances are 1357, 1463, 1591, and $1748 \mathrm{~nm}$, respectively. (b) $\mathrm{Hz}$ mode profile of each mode at the $Y Z$-plane. The azimuthal mode numbers are $9,8,7$, and 6 .

1357 to $1748 \mathrm{~nm}$ with the same hexapole mode as the PC. The resonant wavelength decreases when the azimuthal mode number increases. ${ }^{24}$ Therefore, the hexapole mode excited in the proposed cavity can have four resonant wavelengths distributed from 1357 to $1748 \mathrm{~nm}$ over a broad photonic bandgap spectrum while horizontal electric field profiles are maintained. Here, the index of 2.91 of sphere (index of chalcogenide glass) is chosen for the presented four hexapole modes to be well-localized horizontally.

\section{Spectral Tunability}

When the sphere is chosen with a different radius and refractive index, the hexapole mode can be excited at a desired wavelength ranged in 1339 to $1725 \mathrm{~nm}$, where it covers the entire photonic bandgap of the defect-free PC slab. In order to investigate how the resonant wavelength depends on the index and radius of the sphere, we calculated the wavelength of the hexapole mode with azimuthal mode number 8 (Fig. 3) because the resonant wavelength is in the middle of the bandgap. The resonant wavelength can be controlled from 1343 to $1722 \mathrm{~nm}$ by changing a refractive index of the sphere from 2.43 to 3.16 with $R=1100 \mathrm{~nm}$. The resonant wavelength can also be changed from 1339 to $1725 \mathrm{~nm}$ by increasing the radius of the sphere from 1000 to $1300 \mathrm{~nm}$ with $n=2.67$. A similarly large spectral tuning of $400 \mathrm{~nm}$ is achievable by selecting a proper radius and index of the sphere. On the other hand, each resonant wavelength can be tuned by changing the index or the radius of the sphere.

According to the hexapole mode at the $X Y$-plane and the azimuthal mode with a mode number of 8 at the $Y Z$-plane, the resonant wavelength and $Q$ factor are mapped with respect to the refractive index and radius of the sphere. The resonant wavelength increases when the refractive index or the radius of the sphere increases because the size of the effective resonator becomes larger. There is no resonant mode at the white regions in Figs. 4(c) and 4(d). Since it is over the bandgap from 1309 to $1774 \mathrm{~nm}$, light cannot be confined horizontally in the slab. The $Q$ factor has $3.8 \times 10^{5}$ as the maximum value when the sphere has the refractive index of 1.67 and the radius of $1300 \mathrm{~nm}$. By selecting the index and radius of the sphere, the hexapole mode can be excited with the desired wavelength and $Q$ factors. However, in applications of the optical filter or quantum optics, fine spectral tuning of the cavity mode after fabrication is also required.

In the proposed cavity, the resonant wavelength selectivity accounts for $300 \mathrm{~nm}$ by choosing another azimuthal mode or selecting a sphere with a specific radius and refractive index. After 
(a)

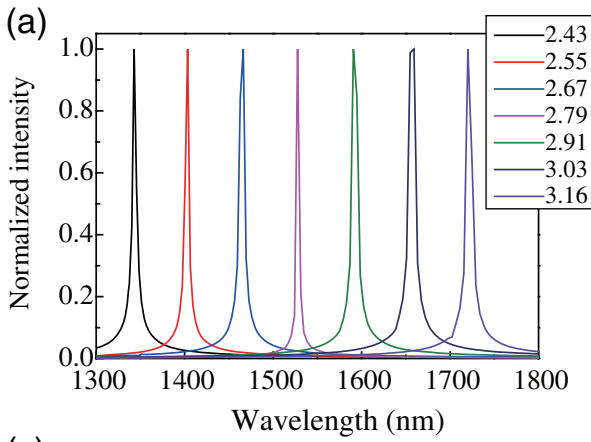

(c)

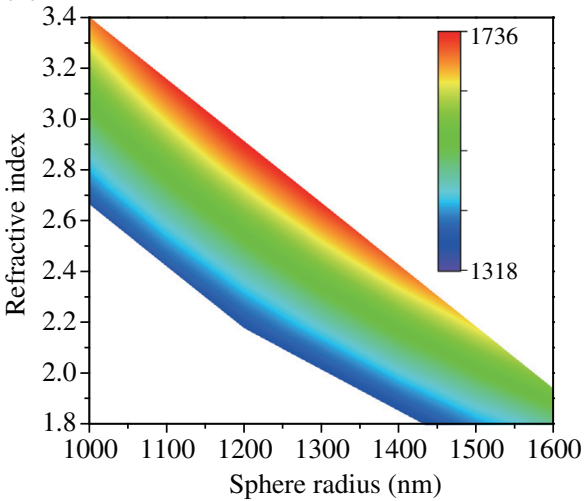

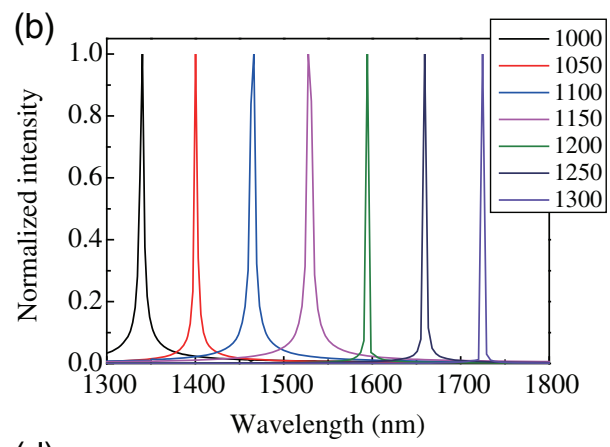

(d)

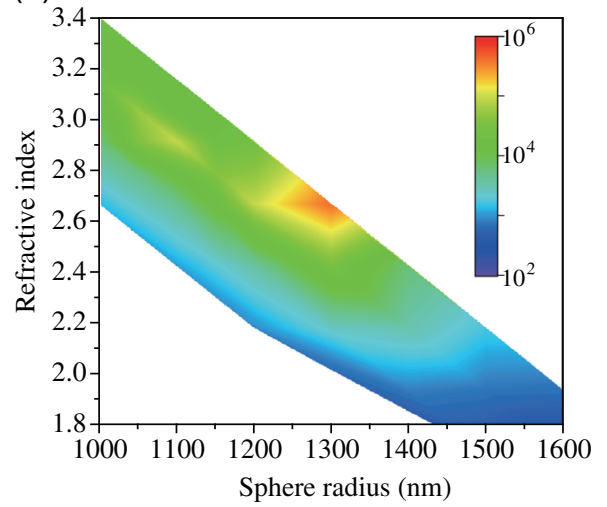

Fig. 4 Resonant peak of the hexapole mode with an azimuthal mode number of 8 versus the (a) refractive index with fixed radius $R=1500 \mathrm{~nm}$ and (b) radius with fixed refractive index of 2.67. (c) 2-D mapping of resonant wavelengths and (d) $Q$ factors with respect to radius and refractive index of microsphere.

(a)

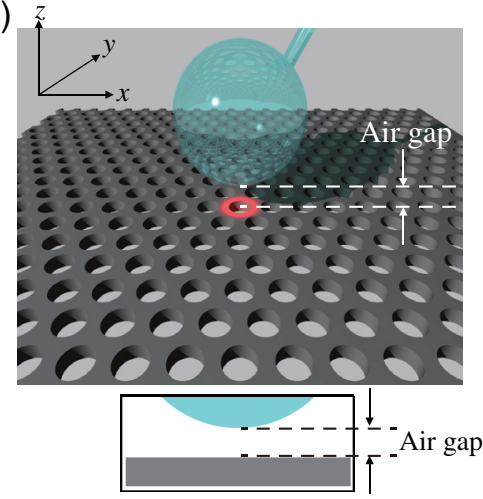

(b)

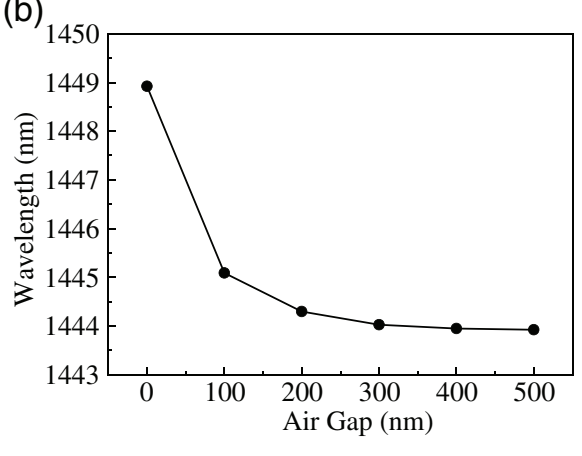

Fig. 5 (a) Schematic of spectral tuning by moving the sphere vertically. The height of the air gap is measured from the surface of the slab to the sphere. (b) Resonant wavelength as a function of the height of the air gap. The wavelength decreases from 1449 to $1444 \mathrm{~nm}$ when the air gap increases from 0 to $500 \mathrm{~nm}$.

the sphere is determined, the resonant wavelength is still finely tunable by adjusting the height of the sphere from the PC slab. In this resonance structure, the effective index can be decreased by inserting an air gap between the dielectric sphere and the PC slab. A resonant wavelength with the sphere that has $R=1000 \mathrm{~nm}$ and $n=2.91$ can be controlled by changing the height of the air gap, as shown in Fig. 5. In fact, the resonant wavelength decreases from 1449 to $1444 \mathrm{~nm}$ when the air gap increases from 0 to $500 \mathrm{~nm}$. The linewidth of the resonance is $0.45 \mathrm{~nm}$ when the air gap is $0 \mathrm{~nm}$ because the $Q$ factor is 3200 . Thus, we can observe the $5 \mathrm{~nm}$ shift induced by changing the air gap because the amount of resonant peak shifting is greater than the linewidth. 


\section{Conclusion}

The PC cavity induced by setting a dielectric sphere on a defect-free PC slab is able to be tuned spectrally as well as relocated spatially. First, the spectral tuning method, by changing the parameters of the sphere such as the radius and refractive index, or by selecting a hexapole mode with a different azimuthal mode number, has a tuning range that covers the entire bandgap from 1309 to $1774 \mathrm{~nm}$. In addition, after selecting a microsphere, adjusting the height of the sphere from the PC slab is a simple method to control the resonant wavelength with a small range of $5 \mathrm{~nm}$ while a high $Q$ of 3200 is maintained. In addition to spectral tuning, by simply moving the microsphere to the desired position on the PC slab, the formation of the high $Q$ PC cavity mode is demonstrated by showing two representative positions: between air holes and at the air hole.

The proposed PC cavity structure can be applied for a wide field. For example, an optical filter with a desired wavelength is simply composed by changing a dielectric sphere between two disconnected PC waveguides. In addition, the PC cavity mode can be employed for efficient manufacturing of a single-photon source because the cavity can be formed at the position where the spatial and spectral overlap with the QD is at a maximum.

On the other hand, the estimation and tolerance analysis of influence of possible deviation for defect free PC and for dielectric sphere in width, radius, position on a slab, and coupling distance on resonant wavelength should be further studied for practical implementation. Deviations of air hole radii and positions in the state-of-the-art PC cavity were reported less than $1 \mathrm{~nm}$, which may give negligible influence in the resonance. ${ }^{25}$ In this letter, $1 \mathrm{~nm}$ and 0.01 deviations of the radius and the refractive index in the sphere induce 1.3 and $5.0 \mathrm{~nm}$ changes in resonant wavelengths, which can be covered by fine tuning. The changes are estimated from the resonant wavelength dependences on the radius and index of the sphere in Fig. 4.

\section{Acknowledgments}

This work was supported in part by the National Research Foundation of Korea (NRF) grant funded by the Korean government (MSIP) (No. NRF-2015R1A2A1A15055998) and in part by the Chung-Ang University Graduate Research Scholarship in 2015.

\section{References}

1. O. Painter et al., "Two-dimensional photonic band-gap defect mode laser," Science 284(5421), 1819-1821 (1999).

2. H.-Y. Ryu, H.-G. Park, and Y.-H. Lee, "Two-dimensional photonic crystal semiconductor lasers: computational design, fabrication, and characterization," IEEE J. Sel. Top. Quantum 8(4), 891-908 (2002).

3. H.-G. Park et al., "Electrically driven single-cell photonic crystal laser," Science 305(5689), 1444-1447 (2004).

4. H.-S. Ee et al., "Ultrasmall square-lattice zero-cell photonic crystal laser," Appl. Phys. Lett. 93(1), 011104 (2008).

5. H.-S. Ee and H.-G. Park, "Ultra-small photonic crystal zero-cell laser cavities," Proc. SPIE 7219, 72190G (2009).

6. M.-K. Seo et al., "Wavelength-scale photonic-crystal laser formed by electron-beam induced nano-block deposition,” Opt. Express 17(8), 6790-6798 (2009).

7. T. Tanabe et al., "All-optical switches on a silicon chip realized using photonic crystal nanocavities," Appl. Phys. Lett. 87(15), 151112 (2005).

8. M.-K. Kim et al., "All-optical bistable switching in curved microfiber-coupled photonic crystal resonators," Appl. Phys. Lett. 90(16), 161118 (2007).

9. P. Michler et al., "A quantum dot single-photon turnstile device," Science 290(5500), 2282 2285 (2007).

10. E. Moreau et al., "Single-mode solid-state single photon source based on isolated quantum dots in pillar microcavities," Appl. Phys. Lett. 79(18), 2865-2867 (2001). 
11. K. Hennessy et al., "Positioning photonic crystal cavities to single InAs quantum dots," Photonic Nanostruct. 2(2), 65-72 (2004).

12. D. Englund et al., "Controlling the spontaneous emission rate of single quantum dots in a two-dimensional photonic crystal," Phys. Rev. Lett. 95(1), 013904 (2005).

13. K. Hennessy et al., "Quantum nature of a strongly coupled single quantum dot-cavity system," Nature 445(7130), 896-899 (2007).

14. F. Intonti et al., "Near-field mapping of quantum dot emission from single-photonic crystal cavity modes," Phys. E Low-Dimens. Syst. Nanostruct. 40(6), 1965-1967 (2008).

15. K. Hennessy et al., "Tuning photonic crystal nanocavity modes by wet chemical digital etching," Appl. Phys. Lett. 87(2), 021108 (2005).

16. J.-Y. Kim et al. "Two-dimensionally relocatable microfiber-coupled photonic crystal resonator," Opt. Express 17(15), 13009 (2009).

17. V. Berger, O. Gauthier-Lafaye, and E. Costard, "Fabrication of a 2D photonic bandgap by a holographic method," Electron. Lett. 33(5), 425-426 (1997).

18. M. Campbell et al., "Fabrication of photonic crystals for the visible spectrum by holographic lithography," Nature 404, 53-56 (2000).

19. S.-H. Kim and Y.-H. Lee, "Symmetry relations of two-dimensional photonic crystal cavity modes," IEEE J. Quantum Electron. 39(9), 1081-1085 (2003).

20. S. G. Johnson et al., "Multipole-cancellation mechanism for high-Q cavities in the absence of a complete photonic band gap," Appl. Phys. Lett. 78(22), 3388-3390 (2001).

21. H.-Y. Ryu and M. Notomi, "High quality-factor whispering-gallery mode in the photonic crystal hexagonal disk cavity," Opt. Express 12(8), 1708-1719 (2004).

22. L. Callot et al., "Very high-Q whispering-gallery mode resonances observed on fused silica microspheres," Europhys. Lett. 23(5), 372-334 (1993).

23. M. Cai, O. Painter, and K. J. Bahala, "Observation of critical coupling in a fiber taper to a silica-microsphere whispering-gallery mode system," Phys. Rev. Lett. 85(1), 74-77 (2000).

24. J.-F. Ku et al., "Whispering-gallery-mode microdisk lasers produced by femtosecond laser direct writing," Opt. Lett. 36(15), 2871-2873 (2011).

25. T. Asano, B.-S. Song, and S. Noda, "Analysis of the experimental Q factors ( $\sim 1$ million $)$ of photonic crystal nanocavities," Opt. Express 14(5), 1996 (2006).

Biographies for the authors are not available. 